Supporting Information

\title{
Liquid Nitrogen-Mediated Thermal Shock for Instantaneous Detachment of Multi-Walled Carbon Nanotube Films from Substrates and Their Application in Supercapacitors
}

\author{
Zhangjun Huang ${ }^{1}$, Haiyan Jia, ${ }^{2}$ Zhaofu Fei, ${ }^{1}$ Paul J. Dyson ${ }^{1 *}$ \\ ${ }^{1}$ Institut des Sciences et Ingénierie Chimiques, Ecole Polytechnique Fédérale de Lausanne (EPFL), CH-1015 \\ Lausanne, Switzerland. \\ ${ }^{2}$ Institute of Mechanical Engineering, Ecole Polytechnique Fédérale de Lausanne (EPFL), CH-1015 Lausanne, \\ Switzerland. \\ *Correspondence to: paul.dyson@epfl.ch
}

Table of Contents

Figure S1. (a) High magnification SEM image of the nMWNT film. HAADF image of (a) low and (b) high magnification of the random stacked MWNTs.

Figure S2. Raman spectra of the nMWNT and MWNT films.

Figure S3. The setup for the CV tests of nMWNT films at different temperatures. 

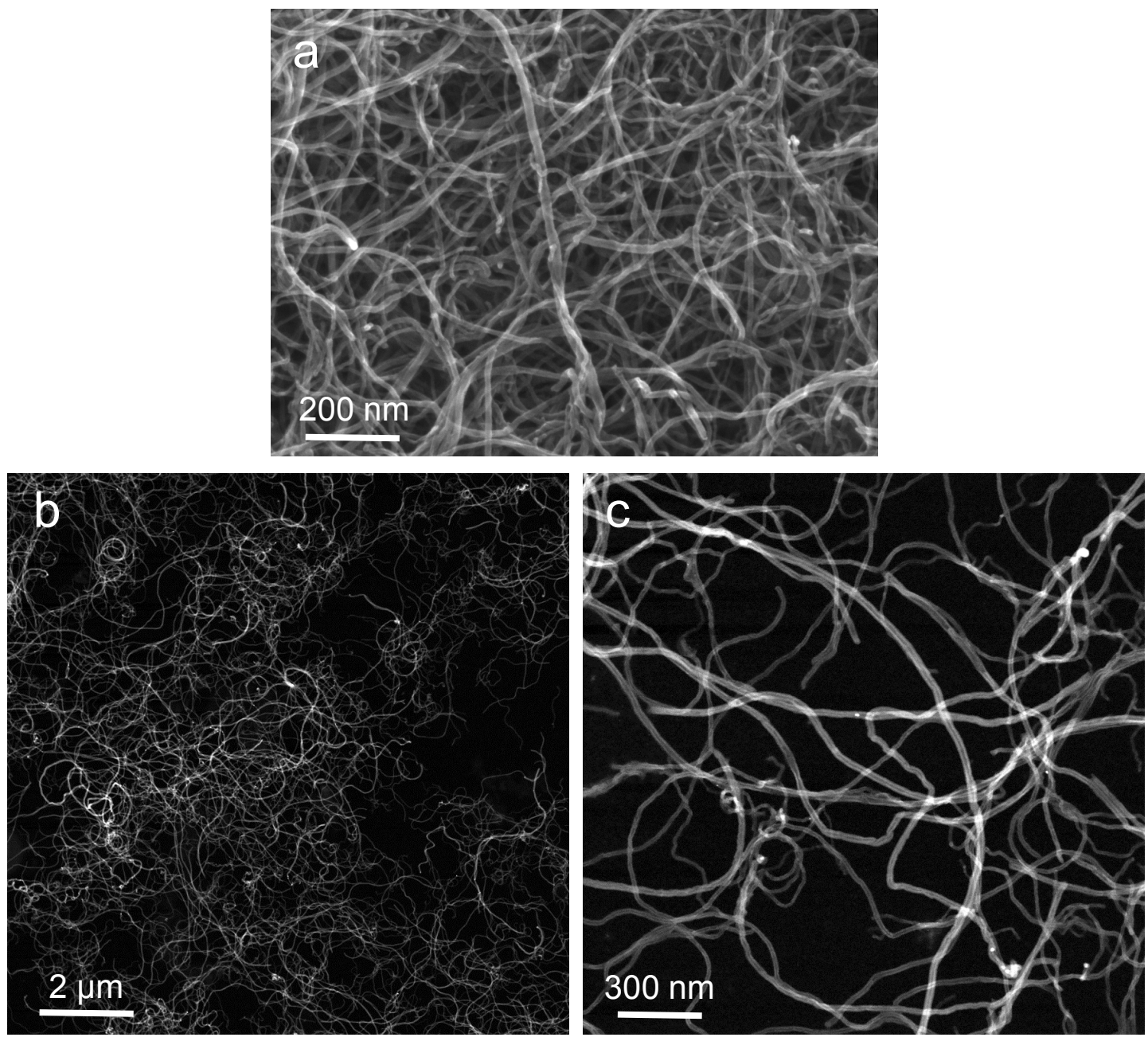

Figure S1. (a) High magnification SEM image of the nMWNT film. HAADF image of (a) low and (b) high magnification of the random stacked MWNTs. 


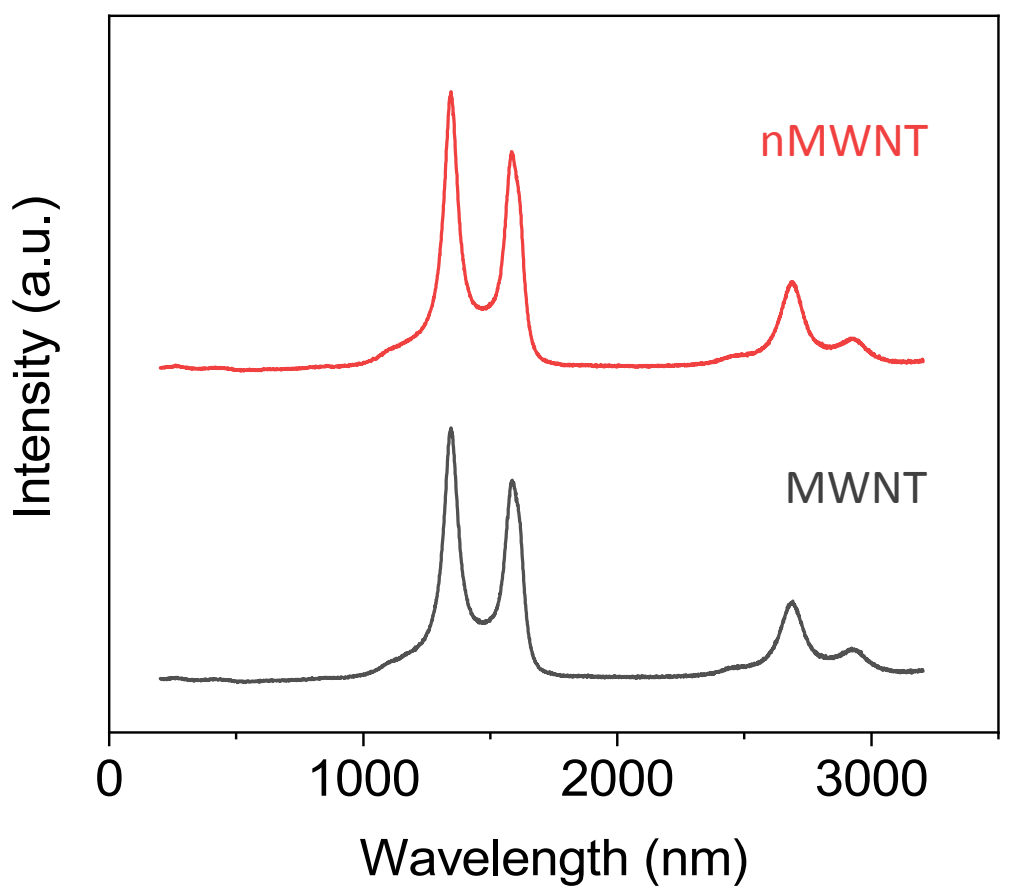

Figure S2. Raman spectra of the nMWNT and MWNT films. 


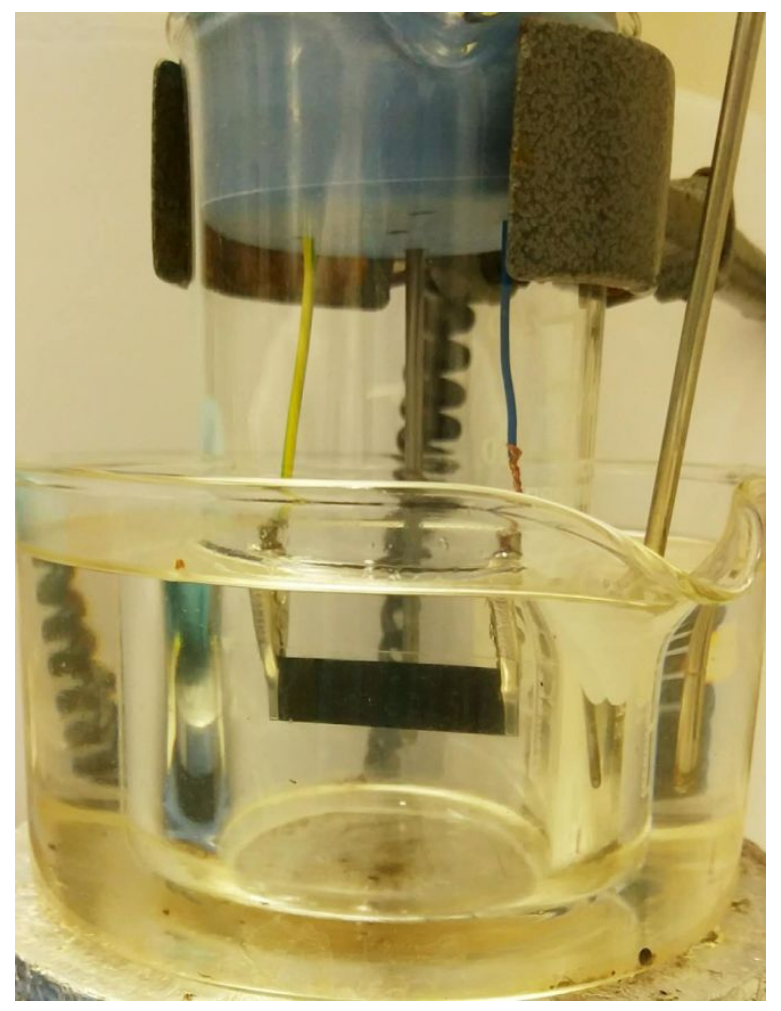

Figure S3. The setup for the CV tests of nMWNT films at different temperatures. 